\title{
Acoustic Propagation and Stability Within an Inviscid, Heat-Conducting Fluid
}

\author{
J. E. McKinney and H. J. Oser \\ Institute for Basic Standards, National Bureau of Standards, \\ Washington, D.C. 20234
}

(January 26, 1970)

\begin{abstract}
Propagation of acoustic waves within a continuum of inviscid, compressible, heat-conducting fluid is evaluated in detail in terms of both frequency (steady-state) and time dependent (transient) functions. The analysis reveals that when the value of the ratio of specific heats, $\gamma$, lies between one and two, the apparent steady-state solutions are conjugate to unstable, or regenerative, "transient" solutions, and, thus, are unacceptable. Propagation is stable for other values $(\gamma=1, \gamma \geqslant 2)$. The common assumption that the steady-state phase velocity varies continuously with increasing frequency from adiabatic to isothermal values is shown to be invalid, except when $\gamma=2$.

Key words: Absorption; acoustic; adiabatic; fluid; heat-conducting; inviscid; isothermal; KirchoffLangevin equation; stability; steady-state; thermal; transient.
\end{abstract}

\section{Introduction}

This paper presents an analysis of the propagation of acoustic waves of small amplitude within a continuum of inviscid, compressible, heat-conducting fluid. The analysis gives the conditions imposed on such a fluid for the propagation to be stable. In this usage "stable" propagation is that manifested by a "stable" fluid, which has no internal energy sources (i.e., a passive fluid). With acoustic propagation the concept of stable propagation implies that for a medium subjected to sinusoidally time-dependent excitation commencing at some time, the response at any point in the medium will approach a bounded steady state. Although the requirement for stable propagation is obvious for any physically realistic fluid (as far as we know), its full significance has apparently been overlooked. In general the inclusion of this requirement will impose a relationship $[1]^{1}$ between certain thermophysical properties of the fluid.

In an earlier paper [1] stability criteria, given as inequalities in terms of certain thermophysical properties, were developed for viscous, heat-conducting fluids, which are a more general class. These criteria were developed without directly analyzing the explicit time dependence of the exact solution. In the following presentation the inviscid fluid, which is simpler to analyze, is examined in detail with respect to both steady-state and transient functions. The analysis reveals some contradictions in present generally accepted concepts with respect to propagation in inviscid fluids. Propagation in these fluids has been assumed to be stable and the steady-state propagation functions have been assumed to vary continuously from adiabatic conditions at low frequencies to isothermal at high frequencies. Our analysis reveals that propagation is unstable when the value of the ratio of specific heats, $\gamma$, lies between one and two, and continuous variation in the steadystate propagation functions from adiabatic conditions at low frequencies to isothermal at high frequencies is not possible in any stable fluid, except when $\gamma=2$. The unstable class of fluids may, of course, be stabilized [1] by the inclusion of viscosity of sufficient magnitude within the model.

Considerable discussion on propagation in heat-conducting fluids has appeared in the literature over the past 100 years, for which some of the more recent examples are given in references [2-9].

Figures in brackets indicate the literature references at the end of this paper. 


\section{List of Symbols}

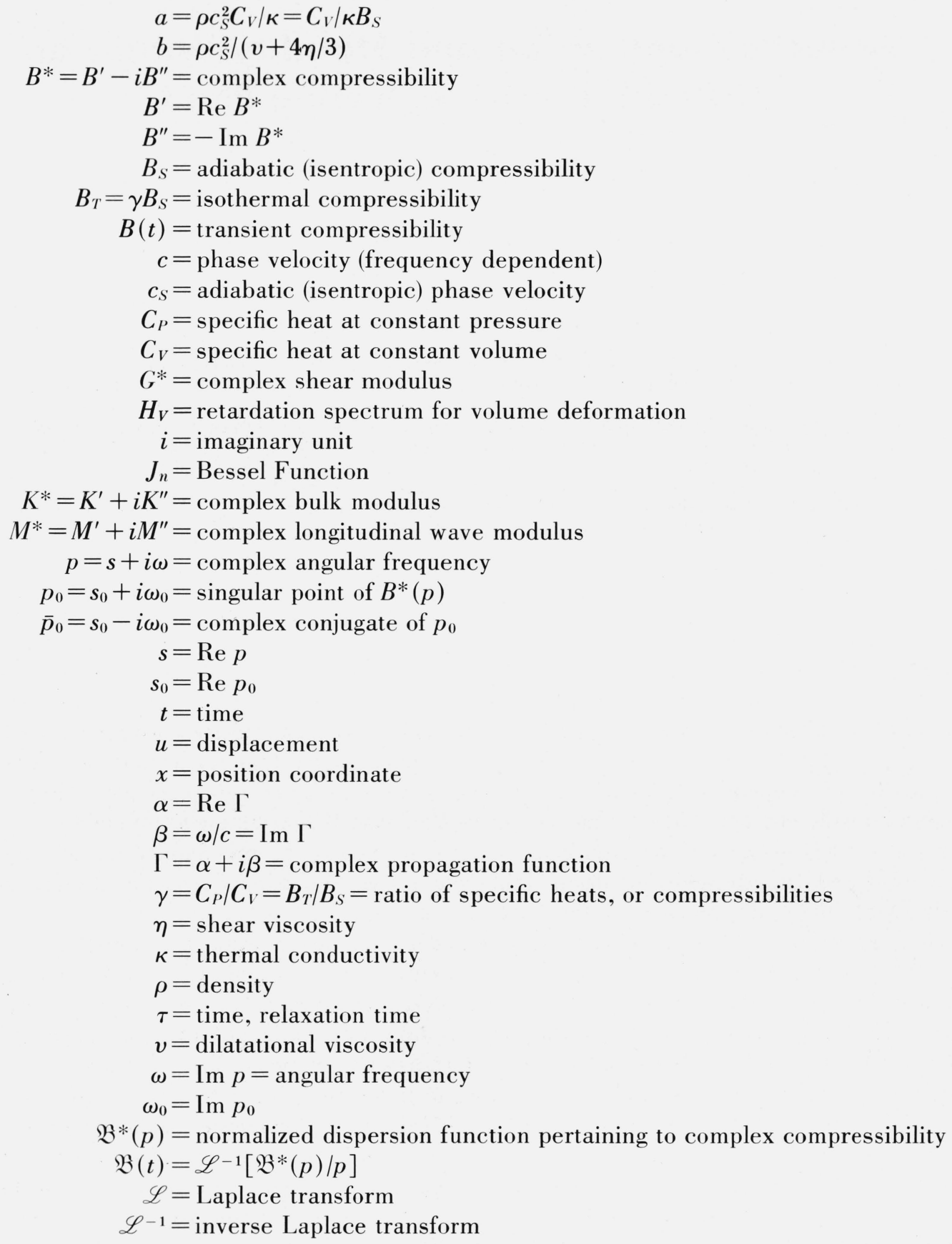


As a result of the complicated algebraic form of the apparent steady-state solutions and the difficulties which arise in obtaining the general solution [9] of the differential equation, the invalid assumptions mentioned in the last paragraph have been widely accepted. In many cases conclusions were based largely on qualitative considerations (for example, references [10-13]) and approximations which facilitate simple calculations, but which shroud other important information. Recently, the analysis has been facilitated by the advent of fast digital computers, which make it a practical matter to trace the branches of the exact propagation functions over a wide frequency range for as many different cases as necessary to illustrate the complete behavior.

\section{Steady-State Functions}

\subsection{Propagation Functions}

In this work we are principally concerned with the forced vibration (sinusoidal) solutions of the differential equation [4] (see appendix),

$$
\frac{\partial}{\partial t}\left[\frac{\partial^{2} u}{\partial t^{2}}-c_{S}^{2} \frac{\partial^{2} u}{\partial x^{2}}\right]=\frac{\kappa}{\rho C_{V}} \frac{\partial^{2}}{\partial x^{2}}\left[\frac{\partial^{2} u}{\partial t^{2}}-\frac{c_{S}^{2}}{\gamma} \frac{\partial^{2} u}{\partial x^{2}}\right]
$$

where $u=u(x, t)$ is the displacement ${ }^{2}$ of an element in the $x$ direction at time $t, c_{S}$ is the adiabatic (or isentropic) phase velocity, $\kappa$ is the thermal conductivity, $\rho$ is the density, taken here in the absence of any disturbance within the medium, $C_{V}$ is the specific heat at constant volume, and $\gamma$ is the ratio of specific heats $\left(C_{P} / C_{V}\right) . c_{S}, \kappa, \rho, C_{V}$, and $\gamma$ are taken to be constants of the fluid independent of $x$ and $t$, which implies that these quantities are independent of the small fluctuations appropriate to the linearized equation (eq (1)). Equation (1) describes the infinitesimal (i.e., linear), uniaxial response of a hypothetical continuum of compressible, inviscid, heat-conducting fluid. This is probably the simplest case for which the role of thermal conductivity is considered in acoustic propagation. For our purposes eq (1) may be taken axiomatically and is used as a point of departure for subsequent arguments.

We are concerned with obtaining the exact solution ${ }^{3}$ of eq (1) subjected to the boundary condition $u(0, t)=u_{0} U(t) \cos \omega t$ (along with other obvious statements with respect to initial conditions and behavior as $x \rightarrow \infty$ ) where $U(t)$ is the unit step function. Although obtaining the Laplace transform, $\mathscr{L}[u(x, t)]=\bar{u}(x, p)$, is straightforward procedure, obtaining its inverse $u(x, t)$ appears to be extremely difficult. However, sufficient information can be obtained from $\bar{u}(x, p)$ to reveal that the exact solution is unbounded, or unstable, when $1<\gamma<2$.

In view of the difficulties in obtaining the exact solution, we will assume the form of the steady-state solution of eq (1). From this we will obtain the frequency-dependent propagation function $\Gamma(p)$ and its equivalent response function $B^{*}(p)$, which appears in simpler form. The stability will be evaluated by applying Laplace transform theory to the response function, and verified later by obtaining the corresponding time-dependent (transient) function.

For waves traveling in the positive $x$ direction only with sinusoidal time dependence, the apparent 4 "steady-state" solutions of eq (1) may be expressed in the following form:

$$
u(x, t)=\operatorname{Re}\left[A e^{p t-\Gamma x}\right]_{s=0}=A e^{-\alpha x} \cos (\omega t-\beta x)
$$

where $p=s+i \omega$ is the complex angular frequency, ${ }^{5}$ and $\Gamma=\alpha+i \beta$ is the complex (frequency dependent) propagation function with $\beta=\omega / c$, where $c$ is the frequency dependent phase velocity.

\footnotetext{
2 The equation for velocity at a point, $v=v(x, t)$, has identical form.

${ }^{3}$ This type (including transient response) of analysis with respect to acoustic propagation is, in general, fairly recent in the literature. For the exact solution pertaining to a viscous, nonconducting fluid, see, for example, reference [14].

+ We speak of eq (2) as an "apparent" steady-state solution inasmuch as it appears to be a steady state and satisfies the differential equation (eq (1)). However, it remains to be seen if eq (2) is the exact solution as $t \rightarrow \infty$.

${ }_{5}^{5}$ The complex frequency, $p=s+i \omega$, is used for the purpose of applying the Laplace transform in the subsequent discussions. It is convenient to use the form $\exp (p t-\Gamma x)$ in lieu of $\exp (\Gamma x-p t)$ in order to facilitate the subsequent use of this transform without modification.
} 
$A$ is a constant (for each branch), which is taken to be real without loss in generality.

Substitution of eq (2) into eq (1) gives the following frequency dependence of the complex propagation function $\Gamma(p)$ :

$$
\Gamma^{2}(p)=\frac{\gamma p^{2}}{2 c_{S}^{2}}\left[1+\frac{a}{p}+\left(1+\frac{2(\gamma-2) a}{\gamma p}+\frac{a^{2}}{p^{2}}\right)^{1 / 2}\right]
$$

where $a=\rho c_{S}^{2} C_{V} / \kappa$, or alternatively, $a=C_{V} / \kappa B_{S}$, remembering $c_{S}^{2}=1 / \rho B_{S}$ where $B_{S}$ is the adiabatic compressibility. Equation (3) may be identified as the propagation function for "steady-state" solution of the Kirchhoff-Langevin equation [8,9] with zero viscosity, or equivalently, with an infinite viscous Reynolds number.

For waves with diminishing intensity in the direction of propagation, as required for passive fluids, $\alpha$ and $\beta$ must have the same sign. This requirement is met for all four branches given by eq (3). The two branches for which $\alpha$ and $\beta$ are both negative are ignored in this analysis because they correspond to backward traveling waves and contribute no additional information to the properties of the fluid. The two branches implied by the $\frac{1}{2}$-power term in eq (3) are commonly called the acoustic and thermal branches. As customary, the acoustic branch is taken to be the one for which the absorption, $\alpha$, vanishes in the low-frequency limit in contrast to an unbounded value in the thermal branch. This choice is consistent with the acoustic branch propagating adiabatically in the low-frequency limit.

Expressions for $\alpha$ and $\beta$ as functions of real frequency are obtained tediously, producing expressions containing eight radicals each, which, indeed, exceed the practical limit of comprehension and space available here. However, the values of these functions with frequency were computed for several examples and are illustrated in section 2.4 along with their corresponding complex response functions.

\subsection{Complex Response Functions}

For mathematical convenience the propagation function obtained in the last section is converted to the complex response function which describes the response to alternating pressure of any small volume element of constant mass within the acoustic field. The information obtained in these two representations is equivalent.

The longitudinal wave propagation function and its corresponding complex response functions are related [15] by

$$
M^{*}=K^{*}+\frac{4}{3} G^{*}=\rho p^{2} / \Gamma^{2}
$$

where $M^{*}=M^{\prime}+i M^{\prime \prime}$ is commonly called the longitudinal wave modulus, which is appropriate to describe linear response for uniaxial strain with sinusoidal time dependence. $K^{*}$ and $G^{*}$ are the complex bulk and shear moduli, respectively. Since we are concerned with inviscid, compressible fluids, which support no shear stress, $G^{*}$ may be taken to be identically zero from which follows

$$
B^{*}=\Gamma^{2} / \rho p^{2},
$$

where $B^{*}=B^{\prime}-i B^{\prime \prime}=1 / K^{*}$ is the complex compressibility. Thus, $B^{*}$ and $\Gamma^{2}$ have the same frequency dependence except for a factor of $p^{2}$. The alternative expressions ${ }^{6}$ equivalent to eq (5) in terms of real and imaginary components at real frequencies are

$$
\begin{aligned}
& B^{\prime}=\left(\beta^{2}-\alpha^{2}\right) / \omega^{2} \rho \\
& B^{\prime \prime}=2 \alpha \beta / \omega^{2} \rho .
\end{aligned}
$$

${ }^{6}$ From inspection of eq (6) it is clear that there are only two distinct branches of $B^{*}(p)$ from the four branches of $\Gamma(p)$ given by eq (3), since $\alpha$ and $\beta$ always have the same sign. 
As commonly used in acoustics (and in this discussion) the complex compressibility relates the dilatation to pressure in both amplitude and phase for sinusoidally time dependent excitation. $B^{*}(p)$ is not a property of the fluid alone, as are the isothermal and adiabatic compressibilities for a viscous fluid, but may also depend on the boundary conditions. Other response functions, such as the characteristic impedance, or admittance, of the medium, could be analyzed in this manner. Since these response functions are equivalent, the conditions for stability obtained through analysis of any of them remain the same. The complex compressibility appears to be the most convenient function for studying this particular case.

The identity,

$$
\sqrt{c+i d}=\sqrt{\frac{1}{2}\left(c+\sqrt{c^{2}+d^{2}}\right)}+i \frac{d}{|d|} \sqrt{\frac{1}{2}\left(-c+\sqrt{c^{2}+d^{2}}\right)}
$$

where $c$ and $d$ may take on all real values, is useful for establishing the inverse relations to eq (6) and certain other useful relations obtained subsequently. In this paper the notation $z^{1 / 2}$ is used for the double-valued function. Expressions of the form $\sqrt{z}$ are interpreted to be single-valued functions with a nonnegative real part. The other branch will be denoted by $-\sqrt{z}$ (with a nonpositive real part). This convention so far does not distinguish the branches of $z^{1 / 2}$ when the value of $z$ is on the negative real axis. For a reason which will become apparent in the treatment of the special case $\gamma=2$ (sec. 4.2), the branch cut is taken along the negative real axis and is assigned the polar argument the value $\theta=-\pi$ (instead of the more customary choice $\theta=\pi$ ). Thus, for the first branch $(-\pi \leqslant \theta<\pi)$ we write $z^{1 / 2}=\sqrt{z}$, which has a nonnegative real part, and for the second branch $(\pi \leqslant \theta<3 \pi), z^{1 / 2}=-\sqrt{z}$, with a nonpositive real part. This choice has the consequence that $\sqrt{-1}=-i$ instead of $+i$ as usual. The form $\pm \sqrt{z}$ or $\mp \sqrt{z}$ is used according to the convention that the upper sign applies to the acoustic branch, whenever applicable.

The inverse relations to eqs (6),

$$
\begin{aligned}
& \alpha=\omega\left[\frac{\rho}{2}\left(-B^{\prime}+\sqrt{B^{\prime 2}+B^{\prime \prime 2}}\right)\right]^{1 / 2} \\
& \beta=\omega\left[\frac{\rho}{2}\left(B^{\prime}+\sqrt{B^{\prime 2}+B^{\prime \prime 2}}\right)\right]^{1 / 2},
\end{aligned}
$$

are readily obtained from eqs (5) and (7).

From eqs (3) and (5) the explicit frequency dependence of the complex compressibility is

$$
B^{*}(p)=\frac{\gamma B_{S}}{2}\left[1+\frac{a}{p}+\left(1+\frac{2(\gamma-2) a}{\gamma p}+\frac{a^{2}}{p^{2}}\right)^{\frac{1}{2}}\right]
$$

which, after taking $p=i \omega(s=0)$, becomes

$$
\begin{aligned}
& B^{*}(\omega)=\frac{\gamma B_{S}}{2}\left[1-\frac{i a}{\omega} \pm \sqrt{1-\frac{a^{2}}{\omega^{2}}-\frac{2 i(\gamma-2) a}{\omega}}\right], 1 \leqslant \gamma<2 \\
& B^{*}(\omega)=\frac{\gamma B_{S}}{2}\left[1-\frac{i a}{\omega} \mp \sqrt{1-\frac{a^{2}}{\omega^{2}}-\frac{2 i(\gamma-2) a}{\omega}}\right], \gamma \geqslant 2 .
\end{aligned}
$$

The following real and imaginary components of $B^{*}(\omega)=\mathrm{B}^{\prime}-i \mathrm{~B}^{\prime \prime}$ are obtained by applying eq (7):

$$
B^{\prime}(\omega)=\frac{\gamma B_{S}}{2}\left\{1 \pm \sqrt{\frac{1}{2}\left[1-\frac{a^{2}}{\omega^{2}}+\sqrt{\left(1-\frac{a^{2}}{\omega^{2}}\right)^{2}+\left(\frac{2(\gamma-2) a}{\omega \gamma}\right)^{2}}\right]}\right\}, 1 \leqslant \gamma<2
$$




$$
\begin{aligned}
& B^{\prime}(\omega)=\frac{\gamma B_{S}}{2}\left\{1 \mp \sqrt{\frac{1}{2}\left[1-\frac{a^{2}}{\omega^{2}}+\sqrt{\left(1-\frac{a^{2}}{\omega^{2}}\right)^{2}+\left(\frac{2(\gamma-2) a}{\omega \gamma}\right)^{2}}\right]}\right\}, \gamma \geqslant 2 \\
& B^{\prime \prime}(\omega)=\frac{\gamma B_{S}}{2}\left\{\frac{a}{\omega} \mp \sqrt{\frac{1}{2}\left[-\left(1-\frac{a^{2}}{\omega^{2}}\right)+\sqrt{\left(1-\frac{a^{2}}{\omega^{2}}\right)^{2}+\left(\frac{2(\gamma-2) a}{\omega \gamma}\right)^{2}}\right]}, \gamma \geqslant 1\right.
\end{aligned}
$$

Equations (10) and (11) are in keeping with our convention given in the last paragraph and consistent with our previously stated choice (sec. 2.1) that the acoustic branch be the one for which the absorption vanish in the limit $\omega \rightarrow 0$. This choice is consistent with $B *(0)=B_{S}$ for the acoustic branch. The two expressions of $B^{*}(\omega)$ and $B^{\prime}(\omega)$ are shown here for the purpose of distinguishing between the acoustic (upper sign) and thermal (lower sign) branches. The reason for the sign reversal at $\gamma=2$ is found in the fact that the roles of the two branches are interchanged in a way which should become clearer during further discussions. Values of $\gamma$ less than unity are not considered because these would require the existence of a negative compressibility or specific heat. It will be shown in the next section that for $1<\gamma<2$, the apparent steady-state solutions are misleading because the fluids are unstable in that the corresponding "transient" functions are regenerative in time. Thus, in excluding the unstable solutions, eqs (10a) and (11a) are applicable only to the special case where $\gamma=1$.

From initial inspection eq (9) appears to have a simple pole at the origin in addition to the branch-point singularities. A binomial expansion of the radical reveals that the simple pole in the acoustic branch disappears by virtue of a cancellation in terms of $a / p$. The presence of the simple pole in the thermal branch is consistent with the unbounded absorption in the low-frequency limit.

\subsection{Evaluation of Stability from the Location of Singularities}

It is commonly assumed in wave propagation that transient solutions decaying with time, are conjugate to steady-state solutions decaying in the direction of propagation. In this section it will be shown that this assumption is not correct in general and is violated for the response functions under consideration when $1<\gamma<2$.

Since the general solution of eq (1) is extremely difficult and apparently has not been obtained, we have chosen an alternate approach, namely, to study the transient ${ }^{7}$ response function $B(t)$, where $B(t)$ is the ratio of time-dependent dilatation, to step function pressure. $B(t)$ corresponds to the response function $B^{*}(p)$ in frequency space. (See eq (9).) The complex and transient functions are related by

$$
B^{*}(p)=p \mathscr{L}[B(t)]
$$

with the corresponding inverse

$$
B(t)=\mathscr{L}^{-1}\left[B^{*}(p) / p\right],
$$

where $\mathscr{L}$ and $\mathscr{L}^{-1}$ are the Laplace and inverse Laplace transform operators. We say a solution of eq (1) is stable if the corresponding transient response function is bounded for all times $t$ after removal of terms linear in $t$. This requires $B^{*}(p)$ to have singularities only on, or to the left of, the imaginary $p$ axis. (See, for example, $[16,17]$.) Terms of $B(t)$ linear in $t$ (corresponding to simple poles of $B^{*}(p)$ at the origin) are permitted, although they are unbounded in the limit as $t \rightarrow \infty$. These terms are analogous to those for steady-flow shear within a Newtonian fluid. In general, for compliances (resulting from step-function stress) of the form $c t^{n}$, the only acceptable values of $n$ for stable response are $0 \leqslant n \leqslant 1 . n<0$ would give a singularity at $t=0$ implying an infinite instantaneous deformation from the application of stress. $n>1$ characterizes a deformation which would continue to increase after removal of the stress (and without bound in the limit $t \rightarrow \infty$ ), as

\footnotetext{
${ }^{7}$ The response to step-function excitation is commonly called the transient response function, in view of the fact that this function usually vanishes at large times. In this case the so-called "transient" response function $B(t)$ often grows with time and is unbounded in the limit as $t \rightarrow \infty$. This behavior is contrary to that implied by the ordinary definition of transient, i.e., existing over only a short time.
} 
can be seen by applying the Boltzmann superposition principle. $0<n<1$ corresponds to a deformation which would recover completely on removal of stress but would increase without limit as long as the stress is applied. $n=0$ and $n=1$ represent purely elastic and purely viscous response, respectively.

In addition to the simple pole at the origin, branch point singularities appear at the values

$$
p=-\frac{(\gamma-2) a}{\gamma} \pm \frac{2 i a \sqrt{\gamma-1}}{\gamma} .
$$

Thus, as required for real time-dependent response functions, singularities off the real axis are always complex conjugates. The locus of these singularities with respect to $\gamma$ is a circle obeying the relation

$$
s_{0}^{2}+\omega_{0}^{2}=a^{2} .
$$

This plot is shown on figure 1 in which the values of $\gamma$ are given for the intercepts. Singularities



Figure 1. Locus of the branch-point singularities of the

complex response function, $\mathrm{B}^{*}(\mathrm{p})$, given by eq $(9)$.

Open circles corresponding to $\gamma=1$ and $\gamma=\infty$ indicate exclusions from the locus.

are restricted to be on and to the left of the imaginary axis when $\gamma \geqslant 2$. When $\gamma$ is exactly one, the radical in eq (9) is a complete square and the point at $p=a$ is regular. However, as soon as $\gamma$ differs from unity (even infinitesimally), a pair of branch points appears and the corresponding instability sets in. Accordingly, the point $\gamma=1$ is an isolated stable point. At $\gamma=\infty$ the branch-point singularities become either simple poles or vanish entirely. (See sec. 4.3.) Accordingly, the points $p=a$ (corresponding to $\gamma=1$ ) and $p=-a$ (corresponding to $\gamma=\infty$ ) are excluded from the locus as indicated by the open circles in figure 1. Since the singularities are paired symmetrically about the real $p$ axis, the transient response will be oscillatory. (See sec. 3.2.) When the singularities are paired about the negative real axis as with $\gamma \geqslant 2$, the oscillations damp out with time. When the singularities are paired about the positive real axis, as they appear for $1<\gamma<2$, the oscillations amplify with time and are unbounded as $t \rightarrow \infty$.

\subsection{Graphical Display of the Functions}

The values of the apparent steady-state functions plotted in this section were obtained in three different ways for the purpose of checking the validity of the results. The functions were programmed in OMNITAB and also in FORTRAN using both the real and complex modes. With OMNITAB and FORTRAN using the real mode, eqs (11) were used to compute the values of the real and imaginary components of the normalized complex response functions. These were converted to the values of the normalized propagation functions using eqs (8) with $\beta=\omega / c$. With FORTRAN using the complex mode eqs (10) were used to compute the values of the normalized complex response function, which were converted to the values of the normalized complex propaga- 
tion function using eq (5) rewritten in the form

$$
\Gamma(\omega)=-\omega \sqrt{\rho B^{*}(\omega)} .
$$

Since we have taken the branch cut of the function $z^{1 / 2}$ in a manner consistent with $\sqrt{-1}=-i$ (see sec. 2.2.), whereas the FORTRAN complex mode assigns the value $i$ to the operation $\sqrt{-1}$, it was necessary to include a statement to make the corresponding inversion at $\gamma=2$ over the range $\omega>a$ (see sec. 4.2.). Figures 2 and 3 give the computed dimensionless complex response functions plotted against dimensionless frequency over the interesting range including the value corresponding to $\omega=a$ for different values of $\gamma$. The principal distinction between these two figures is that figures 2 and 3 pertain to unstable and stable fluids, respectively. Figures 4 and 5 give the corresponding dimensionless propagation functions, which are, of course, more directly realizable experimentally and discussed in the literature. The functions for which the fluids are unstable are included because they are illustrations of functions which have been considered to be stable. Figures 2 and 3 with $\gamma=1.5$ are an example in the range of $1<\gamma<2$ over which the fluids are unstable. The unstable fluids are the only ones (except when $\gamma=2$ ) which would seem to propagate monotonically and continuously between adiabatic and isothermal limits. Figures 3 and 5 with $\gamma=1,2$, and 2.5 are examples for which the fluids are stable. From these figures the nature of the coalescence and inversion of the branches at $\gamma=2$ (see sec. 4.2) is revealed more clearly.

With the exception of the special case of $\gamma=1$, the compressibility always vanishes in the high-frequency limit of the acoustic branch for stable fluids. This corresponds to so-called "anomalous" propagation in that the phase velocity is unbounded in the high-frequency limit. Although the acoustic branch propagates adiabatically in the low-frequency limit, and the thermal branch, isothermally in the high-frequency limit, the functions are never continuous between these limits for stable fluids except when $\gamma=2$. In this case, the infinite derivatives at the value corresponding to $\omega=a$ in all of the functions are consistent with the existence of a branch-point singularity at this value. This singularity also accounts for the coalescence of $B^{\prime}(\omega)$ and $B^{\prime \prime}(\omega)$ in the range $\omega \leqslant a$ and $\omega \geqslant a$, respectively, with $\gamma=2$.

The dotted lines shown with the case for $\gamma=2.5$ were calculated for the components of the viscoelastic resfonse function

$$
B^{*}(i \omega)=\frac{B_{S}}{1+i \omega \tau}
$$

where $\tau$ is the relaxation ${ }^{8}$ time for a single element relaxation spectrum. The limiting values of eq (14) for small and large $p$ are the same as those for eqs (1lb) and (11c) using the upper signs. A single relaxation manifests the narrowest dispersion region possible with respect to any nonnegative relaxation distribution. Accordingly, it is clear from inspection alone of figures $3 \mathrm{c}$ and $5 \mathrm{c}$ that the inviscid functions under discussion cannot be represented by any distribution of relaxation (or retardation) times in the form

$$
B^{*}(p)=B_{g}+\int_{0}^{\infty} \frac{H_{V}(\tau)}{1+p \tau} d \tau
$$

with $H_{V}(\tau)$ nonnegative. For a single relaxation time $H_{V}(\tau)$ is a delta function multiplied by a constant. Since eq (15) applies only to functions with singularities on the negative real $p$ axis, it is not applicable, in general, to the functions under discussion.

${ }^{8}$ This usage is apparently common in acoustic nomenclature. According to viscoelastic nomenclature [18], this quantity should be called a retardation time when used in connection with compliances as with eqs (14) and (15) in contrast to a relaxation time, with moduli. 


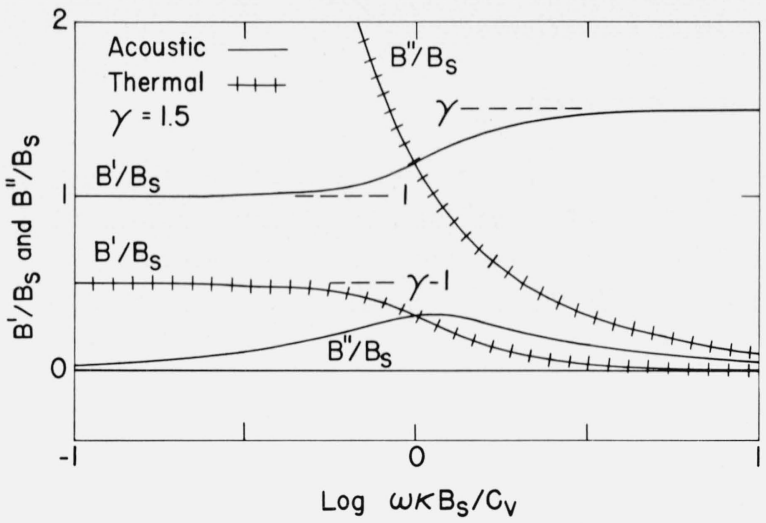

Figure 2. Real and imaginary parts of the normalized complex response functions as functions of normalized frequency with $\gamma=1.5$ as an example in the range of $1<\gamma<2$ for which the propagation is unstable.



(a)

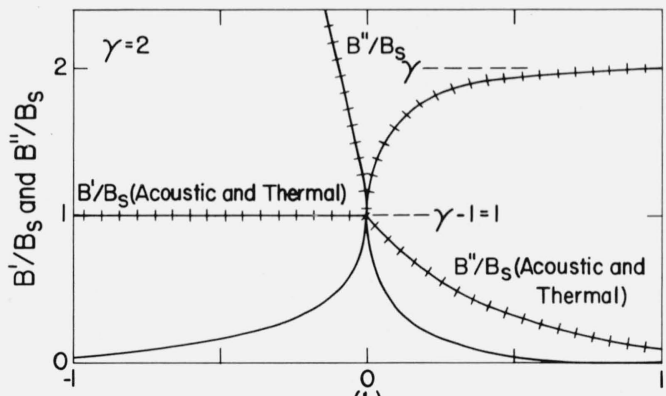

(b)

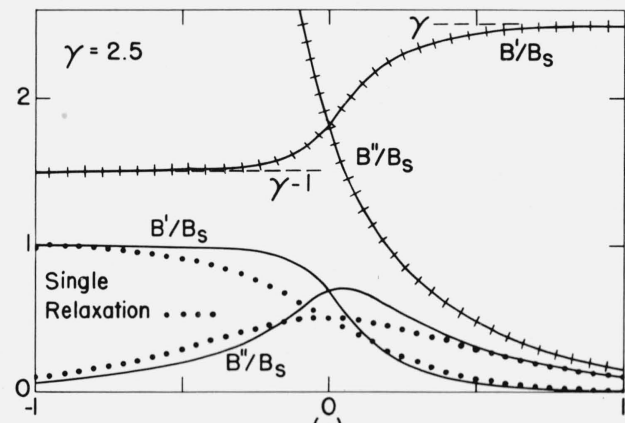

(c)

$\log \omega K B_{s} / C_{v}$

FIGURE 3. Real and imaginary parts of the normalized complex response functions as functions of normalized frequency with (a) $\gamma=1$, (b) $\gamma=2$, and (c) $\gamma=2.5$ as examples at $\gamma=1$, and in the range of $\gamma \geqslant 2$ for which the propagation is stable.

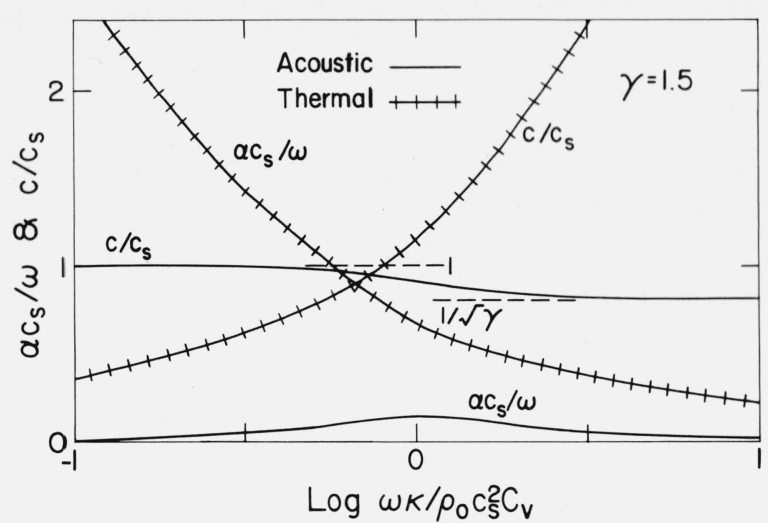

FIGURE 4. Normalized propagation functions corresponding to the functions in figure 2 (unstable propagation).

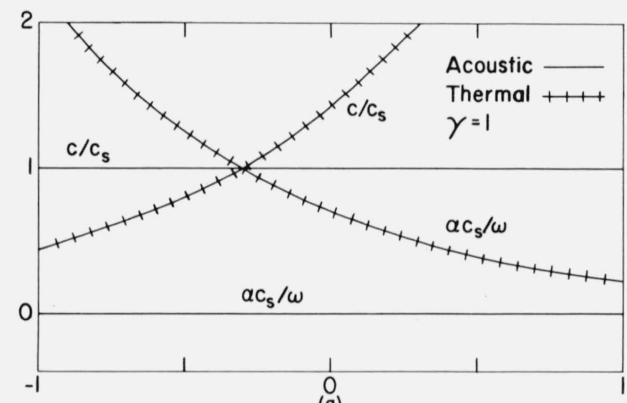

(a)

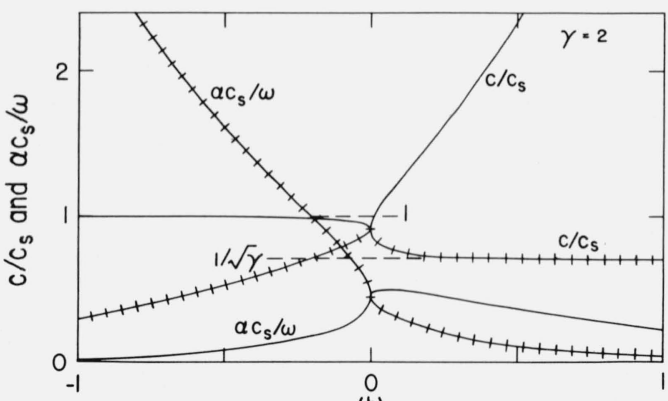

(b)



$\log \omega k / \rho_{0} c_{s}^{2} C_{v}$

FIGURE 5. Normalized propagation functions corresponding to the functions in figure 3 (stable propagation). 


\section{Transient Response Functions ${ }^{9}$}

\subsection{General Integral Formulation}

In the previous paragraphs we have given a necessary and sufficient criterion to distinguish between stable and unstable fluids. For the purpose of obtaining additional insight into the detailed behavior of the solutions, it is useful to study more closely the transient response functions which can be written down explicitly as inverse Laplace transforms of $B^{*}(p) / p$. It is convenient to split the expression for $B^{*}(p) / p$ (see eq $\left.(9)\right)$ as follows:

$$
\frac{B^{*}(p)}{p}=\frac{\gamma B_{S}}{2}\left[\frac{1}{p}+\frac{a}{p^{2}}+\frac{\mathfrak{B}^{*}(p)}{p}\right],
$$

where

$$
\frac{\mathfrak{B}^{*}(p)}{p}=\frac{1}{p}\left[1+\frac{2(\gamma-2) a}{\gamma p}+\frac{a^{2}}{p^{2}}\right]^{\frac{1}{2}} \text {. }
$$

Application of the inverse Laplace transform to eq (16) yields:

$$
B(t)=\frac{\gamma B_{S}}{2}[1+a t+\mathfrak{B}(t)] .
$$

This reduces the problem to finding the explicit time dependence of $\mathfrak{B}(t)$, a function which is double valued, owing to the $1 / 2$-power in $\mathfrak{B}^{*}(p)$.

If we put

$$
\begin{aligned}
s_{0} & =\frac{(2-\gamma) a}{\gamma} \\
\omega_{0} & =\frac{2 a \sqrt{\gamma-1}}{\gamma}
\end{aligned}
$$

and

$$
\begin{aligned}
& p_{0}=s_{0}+i \omega_{0} \\
& \bar{p}_{0}=s_{0}-i \omega_{0},
\end{aligned}
$$

we may write eq (17) as follows:

$$
\frac{B^{*}(p)}{p}=\frac{1}{p^{2}}\left[\left(p-p_{0}\right)\left(p-\bar{p}_{0}\right)\right]^{1 / 2}=\frac{1}{p^{2}}\left[p^{2}-2 s_{0} p+a^{2}\right]^{1 / 2},
$$

which can be decomposed still further into

where

$$
\frac{\mathfrak{B}^{*}(p)}{p}=D(p)-\frac{2 s_{0}}{p} D(p)+\frac{a^{2}}{p^{2}} D(p),
$$

$$
D(p)=\left[p^{2}-2 s_{0} p+a^{2}\right]^{-1 / 2} .
$$

From equation 5.3.34 in [19] we find

$$
\mathscr{L}^{-1}\{D(p)\}=e^{s_{0} t} J_{0}\left(\omega_{0} t\right) .
$$

${ }^{9}$ See footnote 7 . 
Writing \pm to allow for the double-valuedness of the $1 / 2$-power function, we obtain

$$
\begin{aligned}
\pm \mathfrak{B}(t)= & e^{s_{0} \tau} J_{0}\left(\omega_{0} t\right)-2 s_{0} \int_{0}^{t} e^{s_{0} \tau} J_{0}\left(\omega_{0} \tau\right) d \tau \\
& +a^{2} \int_{0}^{t} \int_{0}^{t^{\prime}} e^{s_{0} \tau} J_{0}\left(\omega_{0} \tau\right) d \tau d t^{\prime}
\end{aligned}
$$

Partial integration in the double integral and application of equation 11.3.5 in [20] yields the final result:

$$
\begin{aligned}
\pm \mathfrak{B}(t)= & e^{s_{0} t}\left[\left(1-s_{0} t\right) J_{0}\left(\omega_{0} t\right)-\omega_{0} t J_{1}\left(\omega_{0} t\right)\right] \\
& +\left(a^{2} t-s_{0}\right) \int_{0}^{t} e^{s_{0} \tau} J_{0}\left(\omega_{0} \tau\right) d \tau .
\end{aligned}
$$

Remembering our convention that the upper sign pertains to the acoustic branch, the exact form of the transient response function $B(t)$ for $\gamma \geqslant 2\left(s_{0} \leqslant 0\right)$ is obtained from substituting eq (20) into eq (18):

$$
\begin{aligned}
B(t)=\frac{\gamma B_{S}}{2}\left\{1+a t \mp\left[e^{s_{0} t}\left(1-s_{0} t\right) J_{0}\left(\omega_{0} t\right)-\omega_{0} t J_{1}\left(\omega_{0} t\right)\right.\right. & \\
& \left.\left.+\left(a^{2} t-s_{0}\right) \int_{0}^{t} e^{s_{0} t} J_{0}\left(\omega_{0} \tau\right) d \tau\right]\right\}, \quad \gamma \geqslant 2 .
\end{aligned}
$$

When $1 \leqslant \gamma<2\left(s_{0} \geqslant 0\right)$, eq $(21)$ is valid also except $\mp$ becomes \pm .

\subsection{Approximate Behavior for Large $t$}

Although eq (21) is the general relation applicable for all values of $t$ and $\gamma \geqslant 1$, it is difficult to obtain comprehensible information with respect to the explicit asymptotic behavior as $t \rightarrow \infty$ which is of particular interest here. To obtain this information we take the expansions of $B^{*}(p) / p$ about the three singularities $p=0, p=p_{0}$, and $p=\bar{p}_{0}$. (From the general theory of Laplace transforms [21] the asymptotic behavior of $y(t)$ for large $t$ may be readily determined by the behavior of the leading terms of $\mathscr{L}[y(t)]=y^{*}(p) / p$ expanded about the singularities in the $p$ plane.)

In this connection we are interested in evaluating eq (16) (sec. 3.1) which is

$$
\frac{B^{*}(p)}{p}=\frac{\gamma B_{S}}{2}\left[\frac{1}{p}+\frac{a}{p^{2}}+\frac{\mathfrak{B}^{*}(p)}{p}\right]
$$

where

$$
\frac{\mathfrak{B}^{*}(p)}{p}=\frac{1}{p}\left[1+\frac{2(\gamma-2)}{\gamma p}+\frac{a^{2}}{p^{2}}\right]^{1 / 2} .
$$

Since the inverse transforms of the first two terms in the brackets of eq (16) are readily available, we are only concerned with the appropriate expansions of eq (17).

\section{a. Expansion about $p=0$}

For the expansion about $p=0$, we rewrite eq (17) as follows:

$$
\frac{\mathfrak{B}^{*}(p)}{p}=\frac{a}{p^{2}}\left(1+\frac{2(\gamma-2) p}{a \gamma}+\frac{p^{2}}{a^{2}}\right)^{1 / 2} .
$$


For small $p$ the above equation may be expanded by the binomial theorem indicating double valuedness by the $\mp$ sign.

$$
\frac{\mathfrak{B}^{*}(p)}{p}=\mp\left(\frac{a}{p^{2}}+\frac{\gamma-2}{\gamma p}+\ldots\right)
$$

The dots indicate regular terms which do not contribute to the asymptotic expansions of either $B(t)$ or $\mathfrak{B}(t)$. The corresponding expression for $\mathfrak{B}(t)$, for large $t$, is then

$$
\mathfrak{B}_{0}(t) \approx \mp\left(a t+\frac{\gamma-2}{\gamma}\right)
$$

where the subscript 0 indicates that only the contribution from $p=0$ is included.

\section{b. Expansion about $p=p_{0}$}

In this case we rewrite eq (17) as

$$
\frac{\mathfrak{B}^{*}(p)}{p}=\frac{a}{p^{2}}\left[\left(p-p_{0}\right)\left(p-\bar{p}_{0}\right)\right]^{1 / 2}
$$

which when rewritten in appropriate form for expansion about $p=p_{0}$ becomes

$$
\frac{\mathfrak{B}^{*}(p)}{p}=\left[\frac{a\left(p_{0}-\bar{p}_{0}\right)^{1 / 2}}{p_{0}^{2}} \frac{\left(1+\frac{p-p_{0}}{p_{0}-\bar{p}_{0}}\right)^{1 / 2}}{\left(1+\frac{p-p_{0}}{p_{0}}\right)^{2}}\right]\left(p-p_{0}\right)^{1 / 2}
$$

where all the terms within the brackets are regular at $p=p_{0}$. Consequently,

$$
\frac{\mathfrak{B}^{*}(p)}{p}=\frac{a\left(p_{0}-\bar{p}_{0}\right)^{i / 2}}{p_{0}^{2}}\left(p-p_{0}\right)^{1 / 2}(1+\ldots),
$$

where the omitted terms in the right-hand parenthesis are ignored because they give rise to a series expansion of $\mathfrak{B}(t)$ in decreasing powers of $t$, thus contributing less as $t \rightarrow \infty$. From reference [21] we find

$$
\mathfrak{B}_{1}(t) \approx \mp \frac{a}{2 p_{0}^{2}} \sqrt{\frac{p_{0}-\bar{p}_{0}}{\pi}} e^{p_{0} t} t^{-3 / 2}, \quad t \rightarrow \infty
$$

as the leading term for the contribution from $p=p_{0}$.

\section{c. Expansion about $\boldsymbol{p}=\overline{\boldsymbol{p}}_{0}$}

The expansion about $p=\bar{p}_{0}$ is obtained from the above expression for $\mathfrak{B}_{1}(t)$ by interchanging $p_{0}$ and $\bar{p}_{0}$, which is tantamount to taking the complex conjugate of $\mathfrak{B}_{1}(t)$. Thus,

$$
\overline{\mathfrak{B}}_{1}(t) \approx \mp \frac{a}{2 \bar{p}_{0}^{2}} \sqrt{\frac{\bar{p}_{0}-p_{0}}{\pi}} e^{-\bar{p}_{0} t} t^{-3 / 2}, \quad t \rightarrow \infty
$$

pertains to $p=\bar{p}_{0}$. 


\section{d. Summation of Contributions}

Adding the above contributions $\left(\mathfrak{B}_{0}, \mathfrak{B}_{1}\right.$, and $\left.\overline{\mathfrak{B}}_{1}\right)$ together along with the contributions from the two left-hand terms in eq $(16)$, we obtain the following general $(\gamma \geqslant 1)$ asymptotic expression for $B(t)$ as $t \rightarrow \infty$ :

$$
B(t) \approx \frac{\gamma B_{S}}{2}\left\{1+a t \mp\left[a t+\frac{\gamma-2}{\gamma}+\frac{a}{\sqrt{\pi}} t^{-3 / 2} \operatorname{Re}\left(\frac{\sqrt{p_{0}-\bar{p}_{0}}}{p_{0}^{2}} e^{p_{0} t}\right)\right]\right\} .
$$

Alternatively, the following equivalent expression may be obtained in terms of real quantities:

$$
\begin{aligned}
B(t) \approx \frac{\gamma B_{S}}{2}\left\{1+a t \mp\left[a t+\frac{\gamma-2}{\gamma}+\frac{a}{\left(s_{0}^{2}+\omega_{0}^{2}\right)^{2}} \sqrt{\frac{\omega_{0}}{\pi}}\left(\left(s_{0}^{2}-\omega_{0}^{2}+2 s_{0} \omega_{0}\right) \cos \omega_{0} t\right.\right.\right. & \left.\left.\left.-\left(s_{0}^{2}-\omega_{0}^{2}-2 s_{0} \omega_{0}\right) \sin \omega_{0} t\right) t^{-3 / 2} e^{s_{0} t}\right]\right\},
\end{aligned}
$$

where the upper sign now pertains to the acoustic branch for all $\gamma \geqslant 1$.

For the stable class $\gamma \geqslant 2$ the asymptotic behavior is

$$
B(t) \approx \frac{\gamma B_{S}}{2}\left[1+a t \mp\left(a t+\frac{\gamma-2}{\gamma}\right)\right]
$$

Asymptotes linear in $t$ (although unbounded in the limit $t \rightarrow \infty$ ) are consistent with stable solutions for the reason given in section 2.3. At large $t$ sinusoidal oscillations about these asymptotes are damped by the factor $t^{-3 / 2} e^{s_{0} t}$ for which $s_{0} \leqslant 0$. For the unstable class $1<\gamma<2$ these oscillations regenerate by the same factor for which $s_{0}>0$.

\section{Special Cases for Different Values of $\gamma$}

The following discussion includes the special cases of the functions for different values or ranges of $\gamma$. In these cases the functions may reduce to considerably simpler form and may also reveal distinct characteristics not immediately obvious from the more general form.

\subsection{Stable Case $\gamma=1$}

When $\gamma=1$, the reduced expression for eq (9) becomes

$$
B^{*}(p)=\frac{B_{S}}{2}\left[1+\frac{a}{p} \pm\left(1-\frac{a}{p}\right)\right] ; \gamma=1
$$

Thus, it is apparent that $B^{\prime}=B_{S}$ and $B^{\prime \prime}=0$ for the acoustic branch, and $B^{\prime}=0$ and $B^{\prime \prime}=C_{V} / \kappa \omega$ for the thermal branch. These values are consistent with a constant adiabatic (or isothermal) phase velocity with no absorption in the acoustic branch. The thermal branch propagates in a manner analogous to shear propagation within a Newtonian fluid [2] in that

$$
\alpha=\beta=\sqrt{\frac{\omega \rho C_{V}}{2 \kappa}} .
$$

The steady-state functions for this case are displayed in figures $3 \mathrm{a}$ and $5 \mathrm{a}$.

At $\gamma=1$ the branch point singularities vanish because the square in eq (19) is completed. Accordingly, this point is an isolated stable point and the response is characterized by the simple pole at the origin. 
The following expression for $B(t)$ (which is exact for all $t$ ) is obtained by operating on eq (23) directly:

$$
B(t)=\frac{B_{S}}{2}[1+a t \pm(1-a t)], \quad \gamma=1
$$

It is apparent from the above equation that when $\gamma=1$, the thermal branch (lower sign) of $B(t)$ is analogous to the shear compliance of a Newtonian fluid subjected to a constant stress. Transient terms linear in $t$ (although unbounded in the limit $t \rightarrow \infty$ ) are consistent with stable solutions for the reason given in section 2.3 .

\subsection{Stable Case $\gamma=2$}

When $\gamma=2$, eqs $(11 b)$ and (11c) reduce to

$$
\begin{aligned}
& B^{\prime}(\omega)=B_{S}\left\{1 \mp \sqrt{\frac{1}{2}\left[1-\frac{a^{2}}{\omega}+\left|1-\frac{a^{2}}{\omega^{2}}\right|\right]}\right\} \\
& B^{\prime \prime}(\omega)=B_{S}\left\{\frac{a}{\omega} \mp \sqrt{\frac{1}{2}\left[-\left(1-\frac{a^{2}}{\omega^{2}}\right)+\left|1-\frac{a^{2}}{\omega^{2}}\right|\right]}, \quad \gamma=2 .\right.
\end{aligned}
$$

It is apparent from the above equations that $B^{\prime}$ is single valued and constant over the range $\omega \leqslant a$, and $B^{\prime \prime}$ is single valued over the range $\omega \geqslant a$, in a manner consistent with the branch point singularities at $p= \pm i a$. In this case the two branches of $B^{\prime}$ coalesce for $0 \leqslant \omega \leqslant a$ by virtue of the fact that $(\gamma-1) B_{S}=B_{S}$. The steady-state functions for this case are displayed in figures $3 \mathrm{~b}$ and $5 \mathrm{~b}$.

Since this case corresponds to $d=0$ in eq (7), and because of the single-valuedness mentioned above we have some liberty in designating the branches with respect to acoustic or thermal over the range $\omega>a$. If we had used the customary convention in setting $\sqrt{-1}=i$, in lieu of $\sqrt{-1}=-i$ (see discussion after eq (7), sec. 2.2), the branches (as shown on fig. $3 \mathrm{~b}$ ) would be interchanged over the range $\omega>a$. Since this case has been shown to be stable, we have chosen to deviate from the customary convention. This results in the limiting behavior of $B^{*}(\infty)$ being the same as that for the stable class $\gamma>2$ in that

$$
\begin{array}{ll}
\text { Acoustic } & B^{*}(\infty)=0, \quad \gamma \geqslant 2 \\
\text { Thermal } & B^{*}(\infty)=\gamma B_{S}, \quad 1<\gamma<2 .
\end{array}
$$

The response function $B(t)$ valid for all $t$ (expressed in integral form), and approximate for large $t$, is obtained by setting $s_{0}=0$ in eqs (21) and (22), respectively. The asymptotes are

$$
\lim _{t \rightarrow 0} B(t)=B_{S}[1+a t \mp a t], \quad \gamma=0 .
$$

At large $t$ the sinusoidal oscillations about these asymptotes are damped by the factor $t^{-3 / 2}$. This behavior, which is characterized by the branch points, is distinct from that of undamped resonance, characterized by simple poles also paired symmetrically on the imaginary $p$ axis.

\subsection{Stable Case $\gamma=\infty$}

Since there is no phenomenological bound on the maximum value of $\gamma$, we are considering the role of all possible values for which $1 \leqslant \gamma \leqslant \infty$. (For example, $\gamma$ is apparently singular at the critical point [22] in that $C_{P}$ is more singular than $C_{V}$ or alternatively, $B_{T}$ is more singular than $B_{S}$.) The general form of $B^{*}(p)$ taken from eq (9) in the limit as $\gamma \rightarrow \infty$ is

$$
\lim _{\gamma \rightarrow \infty} B^{*}(p)=\frac{B_{T}}{2}\left\{1+\frac{a}{p} \mp\left[1+\frac{a}{p}-\mathrm{O}\left(\frac{2 a}{\gamma(p+a)}\right)\right]\right\}
$$


where the $\mathrm{O}$ symbol is the one used in asymptotic theory. The value of $B^{*}(p)$ at $\gamma=\infty$ depends on the dependence of $B_{S}$ on $\gamma$ in the limit as $\gamma \rightarrow \infty$. The behavior of $B^{*}(p)$ at $\gamma=\infty$ is summarized in table 1, where the acoustic and thermal branches are treated separately. It is interesting to observe from comparison of eqs (9) and (24) that the branch-point singularities coalesce into a simple pole at $p=-a$ corresponding to $\gamma=\infty$, and, as indicated by table 1 , the simple pole at $p=-a$ vanishes for finite values of $B_{T}\left(B_{S}=0\right)$.

The general form of $B(t)$ in the limit as $\gamma \rightarrow \infty$ corresponding to eq (24) is

$$
\lim _{\gamma \rightarrow \infty} B(t)=\frac{B_{T}}{2}\left\{1+a t \mp\left[1+a t-\mathrm{O}\left(\frac{2}{\gamma}\left(1-e^{-a t}\right)\right)\right]\right\}
$$

The behavior of $B(t)$ at $\gamma=\infty$, is also summarized in table 1. Although $B(t)$ may be unbounded at $\gamma=\infty$ for all values of $t$ under certain conditions as shown, this case is considered to be a stable one because the response does not grow with time after removal of the terms linear in time. A medium of infinite compressibility, for example, a vacuum, does not, necessarily, have intrinsic energy sources as implied by our criterion for an unstable fluid.

TABLE 1. Behavior of $\mathrm{B}^{*}(\mathrm{p})$ and $\mathrm{B}(\mathrm{t})$ at $\gamma=\infty$

\begin{tabular}{l|c|c|c|c}
\hline \multicolumn{1}{c|}{$\lim _{\substack{B_{S} \\
B_{0}}}(\gamma)$} & $B^{*}(p)$, Acoustic & $B^{*}(p)$, Thermal & $B(t)$, Acoustic & $B(t)$, Thermal \\
\hline$B_{S}=0$ & 0 & $B_{T}(1+a / p)$ & 0 & $B_{T}(1+a t)$ \\
$B_{S}=$ Const & $a B_{S} /(p+a)$ & $\infty$ & $B_{S}\left(1-e^{-a t}\right)$ & $\infty$ \\
$B_{S}=(\text { Const })^{\epsilon \gamma}, \epsilon>0$ & $\infty$ & $\infty$ & $\infty$ & $\infty$ \\
\hline
\end{tabular}

\section{Summary of Asymptotic Values and Relations Between Asymptotics of $B^{\star}(p)$ and $B(t)$}

Table 2 summarizes the asymptotics for $B *(p)$ and $B(t)$. The limiting values for $B *(p)$ with $p=0$, and $p \rightarrow \infty$ are taken from the appropriate binomial expansions of eq (9). The values of $B(t)$ with $t=0$, and $t \rightarrow \infty$ are taken from eqs (21) and (22), respectively. The $\pm(\infty)$ notation indicates regenerative oscillations modulated symmetrically about the value zero with the amplitudes going to infinity as $t \rightarrow \infty$.

The following give the relations between the asymptotes of $B^{*}(p)$ and $B(t)$

$$
\begin{aligned}
& B^{*}(\infty)=B(0), \quad \gamma \geqslant 1 \\
& B^{*}(0)=B(\infty), \quad \gamma \geqslant 2, \gamma=1 \\
& B^{*}(0) \neq B(\infty), \quad 1<\gamma<2
\end{aligned}
$$

The first is readily obtained from Laplace transform theory without recourse to any relations given in this paper. The first two are always obeyed in passive response functions. The reason the second is not obeyed in the unstable class $(1<\gamma<2)$ is simply because the rightmost singularities appear to the right of the imaginary axis of the $p$ plane.

TABLE 2. Summary of asymptotics of $\mathrm{B}^{*}(\mathrm{p})$ and $\mathrm{B}(\mathrm{t})$

\begin{tabular}{c|c|c||c|c}
\hline \multirow{2}{*}{} & \multicolumn{2}{|c||}{$\begin{array}{c}\text { Stable class } \\
(\gamma=1, \gamma \geqslant 2)\end{array}$} & \multicolumn{2}{c}{$\begin{array}{c}\text { Unstable class } \\
(1<\gamma<2)\end{array}$} \\
\cline { 2 - 5 } & Acoustic & Thermal & Acoustic & Thermal \\
\hline$B^{*}(0)$ & $B_{S}$ & $B_{S}(\gamma-1+\gamma a / p)$ & $B_{S}$ & $B_{S}(\gamma-1+\gamma a / p)$ \\
$B(\infty)$ & $B_{S}$ & $B_{S}(\gamma-1+\gamma a t)$ & $B_{S} \pm(\infty)$ & $B_{S}(\gamma-1+\gamma a t) \pm(\infty)$ \\
$B^{*}(\infty)$ & 0 & $B_{T}$ & $B_{T}$ & 0 \\
$B(0)$ & 0 & $B_{T}$ & $B_{T}$ & 0 \\
\hline
\end{tabular}

For meaning of $\pm(\infty)$, see text. 


\section{Concluding Remarks}

The analysis of the differential equation for acoustic propagation in a continuum of an inviscid, compressible, heat-conducting fluid yields solutions which display unexpected properties. Although the "apparent" steady-state, forced-vibration solutions correspond to a physically reasonable behavior in that they predict oscillations which always decay in the direction of propagation, the corresponding "transient" response functions predict a response (to step-function excitation) which at long times may oscillate with increasing amplitudes. A regenerative "transient" response implies that the exact solution of the wave equation oscillates with increasing amplitude which is unbounded in the limit of infinite time, and, accordingly, a true steady-state solution does not exist. Since we restrict "acceptable" solutions to those which predict physically realistic behavior for passive, or stable, fluids, we reject the regenerative, or unstable, solutions.

The following statements are considered by the authors to be the most important consequences of the foregoing analysis:

1. Contrary to the popular concept, the phase velocity can never vary continuously with frequency from adiabatic to isothermal values in a compressible, inviscid, heat-conducting fluid, except when the ratio of specific heats is exactly equal to two.

2. Propagation within the above fluid is stable only when either of the following conditions hold:

(i) $\gamma=1$

or

(ii) $\gamma \geqslant 2$.

With the unstable class $(1<\gamma<2)$ the "steady-state" phase velocity of the acoustic branch appears to vary continuously with frequency from adiabatic to isothermal values. With this class the apparent steady-state solution is actually conjugate to a regenerative "transient" solution unbounded in the limit as $t \rightarrow \infty$.

With fluids where the ratio of specific heats lies between the values one and two, stability may be achieved by the inclusion of a minimum viscosity. In a previous paper [1] the stability criteria were developed in terms of thermophysical properties applicable to all values of $\gamma$ in this more general case. These requirements are summarized as follows:

$$
\gamma=1
$$

or

$$
\frac{(2-\gamma) \kappa}{\left(v+\frac{4}{3} \eta\right) C_{P}} \leqslant 1, \quad \gamma>1
$$

where $v$ and $\eta$ are the bulk and shear viscosities. Either of the above conditions is sufficient to insure stability, and these two are the only stable cases.

Although the inviscid fluid is a hypothetical one (since all fluids presumably exhibit a finite viscosity), the unstable fluids cannot be stabilized by the inclusion of a viscosity of infinitesimal magnitude. The results from reference [1] clearly show that a lower bound exists on viscosity to insure stability when $1<\gamma<2$. The above stability requirements were found to be obeyed for all the fluids checked except the molten metals, including mercury, and possibly the superfluids. These results indicate that in some cases different assumptions would have to be made in deriving the propagation equation (Kirchhoff-Langevin equation) for compressible, viscous, heat-conducting fluids.

In general an alternative evaluation of stability may be done by an appropriate qualitative analysis using the Kronig-Kramers [23], or the equivalent Ginzberg [24], relations, which relate the real and imaginary parts of passive complex functions. (The Ginzberg relations apply specifically to velocity and absorption.) In this manner it is often possible to relate the sign of one complex component 
to the sign of the trend of the other. For example, referring to figures 2 and $3 \mathrm{c}$, it is possible to show that $B^{\prime}$ (acoustic) must be monotonically decreasing, and $B^{\prime}$ (thermal), monotonically increasing, with frequency for stable propagation. These trends are not obeyed in figure 2 because corresponding propagation would be unstable. The results with respect to stability are equivalent to those from the foregoing analysis because the Kronig-Kramers relations imply the existence of all singularities on, or to the left of, the imaginary axis of the complex $p$ plane.

The results of this work and those from the more general case [1] indicate the possibility that the apparent steady-state relations obtained from either phenomenological or molecular theories may be inconsistent with stable propagation. In particular, whenever thermoconductivity is included in a model, stability may be questionable. As illustrated here, the fact that the apparent steadystate solutions predict diminishing intensity in the direction of propagation is necessary, but not sufficient, for stable propagation.

\section{Appendix. Derivation of the Propagation Equation}

Equation (1) of the text may be obtained from the hydrodynamic equations [25] applicable to a Newtonian fluid under very general conditions. These equations are the equation of continuity, the Navier-Stokes equation, and the Fourier-Kirchhoff-Neumann energy equation. After dropping viscosity terms and linearizing (replacing $D / D t$ by $\partial / \partial t$, dropping products of derivatives, and taking $\rho$ constant when it appears in a product with a quantity which goes to zero with vainishing motion), these equations for motion in the $x$ direction are

$$
\begin{aligned}
\frac{\partial \rho}{\partial t}+\rho \frac{\partial v}{\partial x} & =0 \\
\rho \frac{\partial v}{\partial t} & =-\frac{\partial P}{\partial x} \\
\rho \frac{\partial e}{\partial t} & =-P \frac{\partial v}{\partial x}+\kappa \frac{\partial^{2} \theta}{\partial x^{2}},
\end{aligned}
$$

where $v, \theta, P$, and $e$ are the velocity, temperature, pressure, and internal energy per unit mass, respectively.

For an inviscid fluid we take $e=e(\rho, P)$ and $\theta=\theta(\rho, P)$ to be single-valued functions of the indicated arguments. From eqs (A.2) and (A.3) the following ordinary thermodynamic relations are implied:

$$
\begin{aligned}
& \left(\frac{\partial e}{\partial P}\right)_{\rho}=\frac{\gamma B_{S} C_{V}}{\alpha P} \\
& \left(\frac{\partial e}{\partial \rho}\right)_{P}=\frac{p}{\rho^{2}}-\frac{\gamma C_{V}}{\rho \alpha_{P}} .
\end{aligned}
$$

Expanding eq (A.3) by the chain rule, we obtain

$$
\rho\left(\frac{\partial e}{\partial \rho}\right)_{P} \frac{\partial \rho}{\partial t}+\rho\left(\frac{\partial e}{\partial P}\right)_{\rho} \frac{\partial P}{\partial t}=-P \frac{\partial v}{\partial x}+\kappa\left(\frac{\partial \theta}{\partial \rho}\right)_{P} \frac{\partial^{2} \rho}{\partial x^{2}}+\kappa\left(\frac{\partial \theta}{\partial P}\right)_{\rho} \frac{\partial^{2} P}{\partial x^{2}}
$$

in which the derivatives operating on $e$ are removed by substituting the above thermodynamic relations, and those on $\theta$, by substituting the definitions

$$
B_{S}=\frac{1}{\rho}\left(\frac{\partial \rho}{\partial P}\right)_{S}=\frac{1}{\gamma \rho}\left(\frac{\partial \rho}{\partial P}\right)_{\theta}, \quad \alpha_{P}=-\frac{1}{\rho}\left(\frac{\partial \rho}{\partial \theta}\right)_{P}
$$


Differentiation of this result with respect to $x$ and $t$ and substitution of eqs (A.1) and (A.2) to replace $\partial P / \partial x$ and $\partial \rho / \partial t$ gives

$$
\frac{\partial}{\partial t}\left[\frac{\partial^{2} v}{\partial t^{2}}-c_{s}^{2} \frac{\partial^{2} v}{\partial x^{2}}\right]=\frac{\kappa}{\rho C_{V}} \frac{\partial^{2}}{\partial x^{2}}\left[\frac{\partial^{2} v}{\partial t^{2}}-\frac{c_{S}^{2}}{\gamma} \frac{\partial^{2} v}{\partial x^{2}}\right]
$$

Equation (1) of the text may be obtained by substituting $\partial u / \partial t$ for $v$. One time derivative may be integrated out by taking a definite integral from $-\infty$ to $t$ and setting $u(x,-\infty)$ and all of its derivatives equal to zero. The result (eq (1) of text) is simply a substitution of $u$ for $v$ in eq (A.4).

\section{References}

[1] McKinney, J. E., and Oser, H. J., J. Acous. Soc. Am. 47, 781 (1970).

[2] Thurston, R. N., in Physical Acoustics, Vol. I-A, Chap. 1, W. P. Mason, Ed. (Academic Press, New York, 1964).

[3] Lindsay, R. B., Mechanical Radiation, p. 266 (McGraw-Hill Book Co., New York 1960).

[4] Vigoureux, P., Ultrasonics, p. 32 (John Wiley \& Sons, New York, 1951).

[5] DeGroot, S. R., and Mazur, P., Non-Equilibrium Thermodynamics, Chap. 12 (Interscience Publishers, New York, 1962).

[6] Perepechko, I. I., Soviet Phys.-Acoustics 10, 284 (1965).

[7] Herzfeld, K. F., and Litovitz, T. A., Absorption and Dispersion of Ultrasonic Waves, Chap. 1 (Academic Press, New York, 1959).

[8] Greenspan, M., in Physical Acoustics, Vol. II-A, Chap. 1, W. P. Mason, Ed. (Academic Press, New York, 1965).

[9] Truesdell, C., J. Rational Mech. Analysis 2, 643 (1953).

[10] Condon, E. U., Am. Phys. Teacher 1, 18 (1933).

[11] Herzfeld, K. F., and Rice, F. O., Phys. Rev. 31, 691 (1928).

[12] Marvin, R. S., and McKinney, J. E., in Physical Acoustics, Vol. II-B, p. 165, W. P. Mason, Ed. (Academic Press, New York, 1965)

[13] Rayleigh, J. W. S., Theory of Sound, Vol. II, p. 28 (Dover, New York, 1945).

[14] Norwood, F. P., J. Acoust. Soc. Am. 44, 450 (1967).
[15] Herzfeld, K. F. and Litovitz, T. A., Absorption and Dispersion of Ultrasonic Waves, Chap. 1, p. 451 (Academic Press, New York, 1959).

[16] Tuttle, D. F., Jr., Network Synthesis, Chap. 4 (John Wiley \& Sons, New York, 1958).

[17] van der Pol, B., and Bremer, H., Operational Calculus, 167-170 (Cambridge, 1959).

[18] Leaderman, H., Trans. Soc. Rheol. 1, 213 (1957).

[19] Bateman, H., Tables of Integral Transforms, Bateman Manuscript Project, California Institute of Technology, Vol. I, A. Erdelyi, Ed. (McGraw-Hill Book Co., New York, 1954).

[20] Luke, Y. L., in Handbook of Mathematical Functions, M. Abramowitz and I. A. Stegun, Eds., National Bureau of Standards Applied Mathematics Series 55 (U.S. Government Printing Office, Washington, D.C. 20402, 1964).

[21] Doetsch, F., Handbuch der Laplace Transformation, Vol. II, Chap. 7 (Birkhäuser Verlag, Basel, 1955).

[22] Egelstaff, P. A., An Introduction to the Liquid State, pp. 159-161 (Academic Press, New York, 1967).

[23] Van Vleck, J. H., in Properties of Short Radio Waves, p. 641, D. E. Kerr, Ed. (McGraw-Hill, New York, 1951).

[24] Ginzberg, V. L., Soviet Phys. Acoustics 1, 32 (1957).

[25] Langlois, W. E., Slow Viscous Flow, p. 58 (MacMillan, New York, 1964).

(Paper 74B2-320) 